\title{
Doppler echocardiographic characteristics of normal and dysfunctioning prosthetic valves in the tricuspid and mitral position
}

\author{
M Pye, N Weerasana, W H Bain, I Hutton, S M Cobbe
}

\begin{abstract}
The Doppler echocardiographic characteristics of 70 prosthetic valves in 35 patients are reported. Twenty nine patients had a Björk-Shiley prosthesis in both mitral and tricuspid positions and six had Carpentier-Edwards valves in both sites. Five of the patients had abnormal tricuspid prostheses on the basis of clinical and echocardiographic criteria. Pulsed wave Doppler echocardiography was used in all examinations. The pressure half times for the normal tricuspid prosthetic valves, 105 (40) ms (BjörkShiley) and 97 (53) ms (CarpentierEdwards), were significantly longer than those of normal mitral prosthetic valves, 75 (18) ms (Björk-Shiley) and 83 (15) ms (Carpentier-Edwards). The range of pressure half times for the abnormal tricuspid valves $(237-530 \mathrm{~ms})$ was distinct from that of the apparently normal tricuspid prosthetic valves (38-197 ms). There was an increase in the peak velocity of the obstructed tricuspid prosthetic valves $(1.69(0.12) \mathrm{m} / \mathrm{s})$ in comparison with normal prostheses $(1.06(0.26) \mathrm{m} / \mathrm{s})$.

The normal range of pressure half times for the Björk-Shiley and Carpentier-Edwards valves in the mitral position is not applicable to the same valves in the tricuspid position. The valve appears to function well with very long pressure half times but a pressure half time of $>200 \mathrm{~ms}$ coupled with a peak velocity of greater than $1.60 \mathrm{~ms}$ without significant valve regurgitation indicates tricuspid obstruction of the tricuspid prosthetic valve.
\end{abstract}

Department of Medical Cardiology, Royal Infirmary, Glasgow

M Pye

I Hutton

S M Cobbe

Department of Cardiac Surgery, Western Infirmary, Glasgow

N Weerasana

W H Bain

Correspondence to DrM Pye, Department of Medical Cardiology, Royal Infirmary, Queen Elizabeth Building, Alexandra Parade, Glasgow G31 2ER.

Accepted for publication 12 September 1989

The detection of prosthetic valve dysfunction often poses a diagnostic challenge because symptoms and signs of dysfunction are often non-specific and can be difficult to detect and measure on clinical examination. The reference standard of investigation for valve function has been cardiac catheterisation (with careful measurement of pressure gradients) and angiography. However, there are practical problems and risks in using invasive methods of assessment, because either the prosthetic valve has to be crossed or direct ventricular puncture attempted in the case of tricuspid prosthetic valves.

It is now widely accepted that Doppler echocardiography provides more detailed and prosthetic valve dysfunction than any other non-invasive procedure. ${ }^{12}$ Although there are many recent studies on the value of Doppler echocardiography in the assessment of mitral and aortic prosthetic valves, ${ }^{1-5}$ there is very little information on the Doppler examination of normal or abnormal tricuspid prosthetic valves ${ }^{67}$ and the numbers reported are small. This study was performed to define a normal range of Doppler indices for the Björk-Shiley tilting disc valve and Carpentier-Edwards xenograft in the tricuspid position and compare them with the same prostheses in the mitral position. We also describe the Doppler characteristics of five clinically dysfunctioning tricuspid prosthetic valves.

\section{Patients and methods}

Thirty five patients were studied; all had undergone mitral and tricuspid valve replacement with the same valve type in both sites. Twenty nine patients had Björk-Shiley (tilting disc) and six had Carpentier-Edwards (xenograft) valve prostheses. Symptoms and clinical and radiographic examinations showed normal mitral prostheses in all patients. In five patients an abnormal tricuspid prostheses was suspected on the basis of the following findings:

(a) Symptoms suggestive of declining cardiac output - for example increasing muscle fatigue on exercise and decline in exercise tolerance with evidence of a significantly raised jugular venous pressure, hepatomegaly, and peripheral oedema.

(b) The absence of significant right or left ventricular dysfunction on echocardiographic examination.

Thirty two of the patients were in atrial fibrillation and three were in sinus rhythm. accurate information on the severity of or the tilting disc. The position of the 
Table 1 Comparison of pressure half times for tricuspid and mitral prosthetic and native valves

\begin{tabular}{lrccc}
\hline & \multicolumn{3}{c}{ Pressure half time (ms) } \\
\cline { 4 - 5 } Valve type & No & Mean $(S D)$ & Median & Range \\
\hline Tricuspid valves: & 24 & $105(40)^{\star}$ & 108 & $50-195$ \\
Normal Björk-Shiley & 6 & $97(53)^{\star}$ & 107 & $38-197$ \\
Normal Carpentier-Edwards & 15 & $67(15)^{\star \star}$ & 69 & $41-71$ \\
Normal native valve & 5 & $353(130)^{\star \star}$ & 308 & $237-570$ \\
Obstructed Björk-Shiley & 29 & $75(18)^{\star}$ & 71 & $71-120$ \\
Mitral valves: & 6 & $83(18)^{\star}$ & 79 & $59-110$ \\
Normal Björk-Shiley & 15 & $(11)^{* * *}$ & 53 & 53 \\
Normal Carpentier-Edwards & Normal native valve & &
\end{tabular}

${ }^{\star} p \leqslant 0.01$, difference between mitral arid tricuspid normal prosthetic valves.

$\star{ }_{p} \leqslant 0.001$, difference between obstructed and normal tricuspid prosthetic valves

$\star \star \star \mathrm{p} \leqslant 0.01$, difference between mitral or tricuspid normal prosthetic and native valves.

transducer was adjusted to obtain the highest maximum velocity with the least velocity dispersion, according to the audio signal and spectral display. The transducer position (apical or parasternal) used to measure the mitral and tricuspid velocities depended on the flow characteristic of the valve. The best alignment with the Doppler beam for native valves and xenografts was often the same because both had central flow characteristics. As expected with the tilting disc valve in the mitral position the major jet was often directed eccentrically towards the interventricular septum. The tricuspid prostheses were orientated with the large orifice opening anteromedially because in patients with considerable right ventricular hypertrophy more conventional posterior opening of the prosthesis could lead to the disc impinging on the subannular posterior wall of the hypertrophied right ventricle. Somewhat modified transducer positions were necessary to obtain optimal and clear spectral recordings in the group with tricuspid prostheses.

To measure peak velocities the maximum peak detected during the examination was recorded over at least 10 cardiac cycles. Atrioventricular pressure half times were recorded over a minimum of seven cardiac cycles and the average of these readings was taken. Pressure half time was calculated by on line computer software as the time taken for the peak velocity to fall to peak velocity $/ 1.4$ according to the method of Hatle et al. ${ }^{8}$ To assess the severity of valve regurgitation, systolic flow in the relevant atrium was studied in all patients by pulsed Doppler mapping of the chamber with small movements of the transducer and shifts of sample volume position from apical and modified parasternal positions. Valve regurgitation was graded according to the depth and width of the systolic jet detected: mild-jet no more than $2 \mathrm{~cm}$ from the valve ring with a jet width $<5 \mathrm{~mm}$; moderate-jet $\leqslant 2 \mathrm{~cm}$ from the valve ring but $>5 \mathrm{~mm}$ wide or jet detected $>2 \mathrm{~cm}$ from the valve ring into the atrium but not reaching the posterior third of the atrium; severe-jet $>5 \mathrm{~mm}$ wide detected in the posterior third of the atrium.

Intraobserver variability was studied by an analysis of the recordings. There was a mean difference of $<5 \%$ for measurements of the same spectral display.

STATISTICAL ANALYSIS

Peak velocities and pressure half times across the mitral and tricuspid prosthetic and native valves were compared by the paired or unpaired $t$ test as appropriate. We used a rank correlation test to compare the peak velocities or pressure half times and time since implantation.

\section{Results}

\section{PRESSURE HALF TIME}

Table 1 shows the results of pressure half times for normal and abnormal prosthetic mitral and tricuspid valves and native valves. There was no significant difference between the pressure half times for prosthetic valves of different sizes in either the mitral or tricuspid position. 'There was no significant difference between the pressure half times of the monostrut or standard Björk-Shiley prostheses.

There was also no correlation between pressure half time and time since implantation in either the mitral or tricuspid positions. The pressure half time was significantly greater in the tricuspid position than in the mitral position for both normal Björk-Shiley and Carpentier-Edwards valves. The pressure half time was significantly longer in the dysfunctioning Björk-Shiley tricuspid valves than in the apparently normal prosthetic valves. Beat to beat variation in pressure half times ranged from 0 to $35 \mathrm{~ms}$ (mean 16) for the mitral valve

Table 2 Comparison of peak early velocity for tricuspid and mitral prosthetic valves

\begin{tabular}{|c|c|c|c|c|}
\hline \multirow[b]{2}{*}{ Valve type } & \multirow[b]{2}{*}{ No } & \multicolumn{3}{|c|}{ Peak velocity $(\mathrm{m} / \mathrm{s})$} \\
\hline & & $\operatorname{Mean}(S D)$ & Median & Range \\
\hline $\begin{array}{l}\text { Tricuspid valves: } \\
\text { Normal Carpentier-Edwards and Björk-Shiley } \\
\text { Normal native valve } \\
\text { Obstructed Björk-Shiley }\end{array}$ & $\begin{array}{r}30 \\
15 \\
5\end{array}$ & $\begin{array}{l}1.06(0.26) \\
0.54(0.11) \\
1.69(0.12)\end{array}$ & $\begin{array}{l}1 \cdot 145^{\star \star} \\
0 \cdot 590^{\star \star \star} \\
1 \cdot 730^{\star}\end{array}$ & $\begin{array}{l}0.6-1.6 \\
0.34-0.94 \\
1.53-1.94\end{array}$ \\
\hline $\begin{array}{l}\text { Mitral valves: } \\
\text { Normal Björk-Shiley and Carpentier-Edwards } \\
\text { Normal native valve }\end{array}$ & $\begin{array}{l}35 \\
15\end{array}$ & $\begin{array}{l}1.17(0.25) \\
0.86(0.32)\end{array}$ & $\begin{array}{l}1 \cdot 200^{\star \star} \\
0.960^{\star \star \star}\end{array}$ & $\begin{array}{l}0.4-1.75 \\
0.4-1.3\end{array}$ \\
\hline
\end{tabular}

${ }^{*} \mathrm{p} \leqslant 0.01$, difference between obstructed and normal tricuspid prosthetic valves.

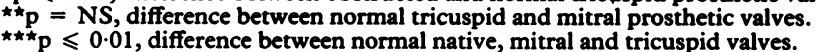


Table 3 Frequency and degree of valve regurgitation

\begin{tabular}{|c|c|c|c|}
\hline & $\begin{array}{l}\text { Valve } \\
\text { regurgi } \\
\text { (No) }\end{array}$ & $\begin{array}{r}\text { itation } \\
(\%)\end{array}$ & $\begin{array}{l}\text { Degree } \\
\text { (mild-severe) }\end{array}$ \\
\hline $\begin{array}{l}\text { Normal Björk-Shiley: } \\
\text { Mitral } \\
\text { Tricuspid }\end{array}$ & $\begin{array}{l}3 / 29 \\
3 / 24\end{array}$ & $\begin{array}{l}10.0 \\
12.5\end{array}$ & $\begin{array}{l}\text { Mild } \\
\text { Mild }\end{array}$ \\
\hline $\begin{array}{l}\text { Normal Carpentier-Edwards: } \\
\text { Mitral } \\
\text { Tricuspid }\end{array}$ & $\begin{array}{l}1 / 6 \\
1 / 6\end{array}$ & $\begin{array}{l}16 \cdot 0 \\
16 \cdot 0\end{array}$ & $\begin{array}{l}\text { Mild } \\
\text { Mild }\end{array}$ \\
\hline $\begin{array}{l}\text { Dysfunctioning Björk-Shiley: } \\
\text { Tricuspid }\end{array}$ & $2 / 5$ & 40.0 & Mild \\
\hline
\end{tabular}

PEAK VELOCITY

Table 2 shows the results for all the peak velocities. There was no significant difference between the peak velocities of normally functioning prosthetic valves in either the mitral or tricuspid position. There was a significant difference between the peak velocity of native mitral and tricuspid valves. The peak velocity of the obstructed valves was significantly higher than in prosthetic tricuspid valves with apparently normal function. Once again there was no significant difference or trend between the peak velocities for different sized valves and no correlation between peak velocity and time since implantation in either position.

\section{VALVE REGURGITATION}

Table 3 shows the results of the regurgitation study. Valve regurgitation was more common in the normal Carpentier-Edwards valves than in the normal Björk-Shiley valves. There was a significantly higher incidence of regurgitation in the five patients with dysfunctional valves but in all cases this was mild.

\section{Discussion}

The normal value for pressure half time across the native mitral valve was reported to be $<60 \mathrm{~ms}^{8}{ }^{8}$ but surprisingly there are no data on normal pressure half times for the native tricuspid valve. There is very little published information on the Doppler indices for normal or abnormal native or prosthetic tricuspid valves. ${ }^{5-7910}$ These studies of small numbers of normally functioning tricuspid xenografts reported pressure half times ranging from 67 to $260 \mathrm{~ms}$. There are few data on tricuspid prostheses because there is no satisfactory method of recording right ventricular pressure and hence mean pressure gradients without transthoracic puncture of the right ventricle or crossing the prosthetic valve with a catheter.

Both mechanical and biprosthetic valves cause some obstruction of blood flow even when they function normally and this is reflected by our finding that the pressure half times and peak velocities in our study exceeded the normal values for native valves in the mitral and tricuspid positions. A major advantage of this study was that each patient had the same type of prosthetic valve in both the mitral and tricuspid position. It was therefore possible to study the behaviour of the valve in different positions at the same examination, and thus under virtually identical haemodynamic conditions.
We found no significant difference in the pressure half time or peak velocity between the Björk-Shiley or Carpentier-Edwards valve implanted in the same valve position. However, the pressure half time range for normal tricuspid prosthetic valves was found to be considerably longer than that of mitral prosthetic valves (Björk-Shiley 50-195 ms $v$ 71-120 ms and Carpentier-Edwards 38-197 ms $v$ 79-110 $\mathrm{ms}$ respectively). This could represent functional obstruction to flow caused by the nature of the valve coupled with greater tissue ingrowth or pannus formation on the tricuspid valve. However, the tricuspid prosthesis seems to function satisfactorily even with what would be considered a very long pressure half time of $197 \mathrm{~ms}$. It is worth emphasising that the dividing range between clinically normal and abnormal prosthetic tricuspid valves is small (197-237 ms). For mitral prosthetic valves a gradual increase in obstruction to flow might be expected to present earlier as dyspnoea on effort and evidence of pulmonary venous congestion. Perhaps the tricuspid valve has to be more critically obstructed before symptoms or signs are apparent. Once this critical point is reached the valve dysfunction quickly becomes apparent, possibly owing to added thrombus formation over an area of low flow. Although pressure half time is predominantly dependent on the valve area it is by no means the only factor of influence. Flow deceleration, of which pressure half time is one measure, across normal mitral and tricuspid native valves may increase with impairment of left or right ventricular diastolic function. ${ }^{11} 12$ Patients who undergo simultaneous mitral and tricuspid valve replacement often have more severe, and less reversible, pulmonary hypertension. This could conceivably lead to right ventricular hypertrophy, impairment of right ventricular diastolic function, and misleading long pressure half times. Although there was no significant impairment of right or left ventricular systolic function on echocardiographic examination in our patients, this does not exclude some degree of abnormal diastolic function.

In our group mitral valve pressure half times showed only mild beat to beat variation, even in atrial fibrillation (0-35 ms) while there was considerable beat to beat variation across the tricuspid prosthetic valves (10-95 ms). This has been noted by other groups ${ }^{5}$ and probably represents more considerable changes in flow and valve orifice area with respiration and varying cardiac cycle length. This emphasises the importance of taking pressure half time measurements from several cardiac cycles especially for tricuspid prosthetic valves. The peak flow velocities across both types of normal tricuspid prosthetic valve were similar, but the five obstructed tricuspid valves showed a significant increase in peak velocity, ranges $0.6-$ $1.6 v 1.53-1.94 \mathrm{~m} / \mathrm{s}$ respectively. Interestingly there was no significant difference in peak velocities between mitral or tricuspid prosthetic valves.

Unfortunately all flow mapping techniques with either colour flow or standard pulsed 
Doppler have only a limited relation to the degree of valve regurgitation. Many of the limitations are similar to those found with contrast ventriculography - that is the size and pressure of the chamber into which regurgitant blood is flowing influence the contrast ventriculographic appearances of the regurgitant dye and also the size of the regurgitant Doppler signal. So we may have missed an eccentrically placed leaking prosthetic jet with pulsed Doppler, and metal valves can create reverberations that mask flow behind the prostheses, leading to blind spots when the transthoracic approach is used for the pulsed Doppler sampling technique. Regurgitation was more common in the group with obstructed valves but in all this was judged to be mild, and this degree of regurgitation should not have interfered with the pressure half time or peak velocity results.

We conclude that long term follow up of patients with prosthetic valves is required to assess the full role of Doppler echocardiography in detecting and measuring dysfunction of the tricuspid prosthetic valve. The normal range of pressure half times for the BjörkShiley and Carpentier-Edwards valves in the mitral position cannot be applied to valves in the tricuspid position. The valve seems to function well with very long pressure half times and we would be cautious about diagnosing valve obstruction/dysfunction on the basis of a prolonged pressure half time or increase in peak velocity alone. However, there may be a narrow dividing line between normal and abnormal tricuspid prosthetic valves and once this is passed valve dysfunction may quickly become apparent. Our study showed that a pressure half time of $>200$ ms coupled with a peak velocity of $>1.6 \mathrm{~m} / \mathrm{s}$ in the absence of severe regurgitation confirmed appreciable obstruction of the tricuspid prosthetic valve. We recommend that all patients with prosthetic valves should be examined by Doppler echocardiography soon after operation. Such a baseline characterisation of each individual's Doppler values would make serial evaluation much more meaningful.

1 Williams GA, Labovitz AJ. Doppler haemodynamic evaluation of prosthetic (Starr-Edwards and BjörkShiley) and biprosthetic (Hancock and CarpentierEdwards) cardiac valves. Am J Cardiol 1985;56:325-32.

2 Hatle L, Angelson B. Doppler ultrasound in cardiology physical principles and clinical applications. Prosthetic valves. Philadelphia; Lea and Febiger, 1985:188-205.

3 Pandis IP, Ross J, Mintz GS. Normal and abnormal prosthetic valve function as assessed by Doppler echocardiography. J Am Coll Cardiol 1986;8:317-26.

4 Ramirez ML, Wong M. Reproducibility of stand alone continuous wave Doppler recordings of aortic flow velocity across biprosthetic valves. Am J Cardiol velocity across

5 Gibbs JL, Wharton GA, Williams GJ. Doppler echocardiographic characteristic of the Carpentier-Edwards xenograft. Eur Heart J 1986;7:353-6.

6 Wilkins GT, Gillam LT, Kritzer GL, Levine RA, Palacross IF, Weyman AE. Validation of continuous wave Doppler echocardiographic measurements of mitral and tricuspid prosthetic valve gradients: a simultaneous Dopplercatheter study. Circulation 1986;74:786-95.

7 Alam M, Rosman HS, Lakier JB, et al. Doppler and echocardiographic features of normal and dysfunctioning biprosthetic valves. J Am Coll Cardiol 1987;10:851-8.

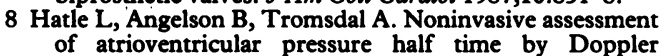
ultrasound. Circulation 1979;60:1096-104.

9 Dennig K, Kraus F, Rudolph W. Doppler-echokardiographische bestimung des schweregrades der trikuspidalstenose. Herz 1986;11:332-6.

10 Wilson N, Goldberg SJ, Dickinson DF, Scott O. Normal intracardiac and great artery blood velocity measurements by pulsed Doppler echocardiography. Br Heart $J$ by pulsed Dop

11 Spirito $P$, Maron BJ. Doppler echocardiography for assessing left ventricular diastolic function. Ann Intern Med 1988; 109:122-6.

12 Isobe $M$, Yazaki $Y$, Takaku $F$, et al. Right ventricular filling detected by pulsed Doppler echocardiography during the convalescent stage of inferior wall acute myocardial infarction. Am J Cardiol 1987;59:1245-50. 